\title{
NUMERICAL MODELING OF A PHASE CHANGE MATERIAL THERMAL STORAGE TANK FOR SOLAR HEATING SYSTEM
}

\author{
Olakunle Isamotu, Olawale Okegbile \\ Department of Mechanical Engineering \\ Federal University of Technology Minna, Niger State, Nigeria
}

\begin{abstract}
With a view to finding an improved thermal storage capability of solar water tank, the thermodynamics of a thermal storage tank with a eutectic phase change material was studied. The thermal exchange between the heat transfer fluid (HTF) and the PCM were investigated. The PCM are helpful in solar-systems for helping to satisfy the energy-demand when alternative energy isn't obtainable. The solar-water heaters based on phase change materials (PCMs) have the advantage of high storagedensity at a low temperature range. However, there are some simplifying assumptions that were established during this paper. Underneath simplifying assumptions, it's shown that the governing equations are the three energy conservation equations written for the thermal transfer fluid, the tank and the PCM. The PCM energy conservation is written in-terms of physical-property (enthalpy). All of the differential equations are numerically resolved in line with a finite distinction scheme technique. Simulations are performed to derive the temperature profiles in the PCM storage tank region at the solar water heater. Several simulation runs are created for ambient-conditions by varying the water temperature of the heat transfer fluid and finally, the simulated and the experimental results was additionally created for optimum comparison.
\end{abstract}

Keywords: Thermal storage, Solar heating, Phase Change Material, Analytic approximate solution, Experimentalvalidation

\section{INTRODUCTION}

The growing variety of issues with the world's dependence on fossil fuels continues to foster the public's want to pursue clean, renewable sources of energy. The last decades had experience a rise in energy demand, along with a growing awareness of the limitation of emissions of greenhouse gases and pollutants that has resulted during a vital impulse to the event of technologies aimed toward energy saving and the assembly of energy from renewable sources. The InterGovernmental Panel on temperature change (IPCC) report [1] shows that human elementary role on climate change attributable to carbondioxide emissions from energy consumption. The utilization of renewable energy sources and enlarged energy efficiency are the most methods to scale back the dependency of fossil fuels and dioxide emissions. However, because it is understood, that renewable energy sources like solar and wind energy particularly, aren't solely characterized by discontinuous accessibility but are however stricken by random variations due to climatic conditions.

Green researches everywhere the planets are into deep search of latest and non-conventional energy sources. One in every of the choices they came up with was to develop energy storage devices, while rooting for different new sources of energy. Thermal energy storage gave us the solution as a result of this, this method has the potential to realize energy savings that successively minimises the surroundings impact associated with non- renewable energy use. However, these systems give a long-lasting answer for correcting the anomalies that's usually found between the provision and demand of energy. Heat storage may be a comparatively new space of study and pioneered by Dr. Telkes within the 1940's. Though' within the time past it received the abundant required attention throughout the energy crisis. During the mid-1970 and early 1980 it had been extensively researched to be used in solar heating systems. Once the energy crisis subsided, less stress was placed on heat storage that has brought us back to wherever we are at first. Though' analysis into heat storage for solar heating systems continues to associate in nursing extent, and recently it's progressively finding its method into the advanced analysis area. There has been a gradual and continuous accomplishment within the space of waste heat recovery, building energy conservation, air conditioning applications and cargo leveling for power generation. As demand for air heating and cooling enlarged greatly throughout the last decade, giant demands of electrical power and restricted reserves of fossil fuels have led to a surge in interest with regard to energy efficiency. Energy usage differs considerably throughout the day and night per the demand by industrial, business and residential activities. In hot and cold climate countries, the major part of the load variation is due to air conditioning and domestic space heating respectively. Perhaps, this differential results in a differential system for peak and off peak periods of energy consumption. Higher 
power generation, distribution management and vital economic profit will be achieved if a number of the height load might be shifted to the off peak load amount. This could be achieved by thermal energy storage for heating and cooling in residential and business building institutions. Heat storage (LHS) can be accomplished through solid-liquid, liquid-gas, solid-gas, and solid-solid section transformations, however this study is proscribed to solid-liquid and solid-solid section. Temperature-time diagram for the heating of a substance as shown in Figure 1 presents a rise of internal energy, once energy within the variety of heat is added to a substance. The well-known consequence is an increase in temperature (sensible TES) or change of phase (latent TES). Beginning with an initial solid state at origin $\mathrm{O}$, addition of heat to the substance initially causes solar-heating of the solid (region $\mathrm{O}-$ A), followed by a solid-to-liquid action (region A-B), a sensible-heating of the liquid (region $\mathrm{B}-\mathrm{C}$ ), a liquid to vapour state (region $\mathrm{C}-\mathrm{D}$ ), and a sensible-heating of the vapour (region D-E). The total-amount of heat will be written in the following formula [2].

$$
\begin{aligned}
& Q=m\left\{\int_{T_{0}}^{T_{A}} C_{p s}(T) d T+q_{t}+\int_{T_{E}}^{T_{C}} C_{p l}(T) d T+\right. \\
& \left.q_{i}+\int_{T_{D}}^{T_{E}} C_{p v}(T) d T\right\}
\end{aligned}
$$



Figure 1. Temperature-time diagram for the heating of a substance [2]

It is seen from the graph that the actual solid substance changes its state consecutively from solid to vapour (phase change) with increase in temperature. A solid-solid system also shows abundant promises.

\section{Descriptions of Phase Change MATERIALS}

Phase change materials (PCM) are "Latent" heat storage materials. The thermal energy transfer happens when a material changes from solid to liquid, or liquid to solid. This can be referred to as a change of state, or "Phase." at first, the solid-liquid PCMs perform like typical storage materials; their temperature rises as they absorb heat. In contrast to typical (sensible) storage materials. PCM absorbs and unleash the absorbed heat at a virtually constant temperature. They store 5-14 times additional heat per unit volume than sensible storage materials like water, masonry, or rock. An outsized variety of PCMs are known to melt with a heat of fusion in any required range. However, for his or her employment as heat of transformation storage materials they must exhibit some certain desirable thermodynamic, kinetic and chemical properties [3]. Moreover, economic concerns and simple convenience of those materials must be unbroken in mind. The PCM utilized in the look of thermal-storage systems passes the fascinating thermo- physical, mechanics and chemical properties [4]. [5] Developed a mathematical and experimental model for the charging method of a solar dish storage system integrated with salt hydrate as a PCM. They performed their experimental trials underneath the winter weather of Israel. the heat- energy hold on was discharged to cold water flowing in an exceedingly finned device (HX) situated within a layer of stationary heat transfer fluid (HTF). In the mathematical model, the researchers used the enthalpy-based finite distinction methodology and unnoticed the result of convection. They assumed one-dimensional transient heat physical phenomenon within the PCM and stationary heat transfer layer. The projected model showed high correct validation with the experimental PCM temperature measurements. However, the neglect of the convection heats transfers within the stationary heat transfer reduced the accuracy of their mathematical predictions. [6] Projected a comparative experimental study, underneath a typical sunny day in Asian nation, to research the likelihood of enhancing the performance of a flat-plate solar dish and storage-tank by group action wax with a melting temperature of $60.45^{\circ} \mathrm{C}$ directly involved with the collector's absorbent material plate. The projected system was studied and compared with the standard collector at three totally different inclination angles together with $10^{\circ}, 20^{\circ}$, and $30^{\circ}$ and constant water mass rate of flow of $0.5 \mathrm{~kg} / \mathrm{min}$. The results showed that the mixing of PCM raised the thermal absorption to $71.15 \%$ and reduced the thermal heat losses to be below $4.34 \mathrm{~W} / \mathrm{m}^{2} \mathrm{~K}$. The projected collector and storage tank achieved associate efficiency of $52 \%$ at an inclination angle of $10^{\circ}$, which was higher by $23 \%$ over the standard collector.

Researcher [7] conducted studies on a solar hot-water tank storage collector system. The method incorporates a flat plate collector manufactured from galvanized steel tubes hooked up to associate Aluminum plate with MAXORB selective surface and a double walled one hundred litres capability galvanized steel vessel. Non-conductor insulation was but wont to insulate the vessel. The vessel was settled up gradient from the collector. This method exclusively depends on natural circulation to drive the recent water into the overhead vessel and permits for cold water to re-circulate to the collector just as the normal return and supply pipes. This investigator discovered that the system will generate about $50.6^{\circ} \mathrm{C}$ water at $4: 00 \mathrm{pm}$ and $41.6^{\circ} \mathrm{C}$ water the subsequent morning. The potency of this method was calculated to be forty-seven. within the course of his study, the investigator discovered that the value of a system with a galvanized steel Aluminum 
collector valued $(\$ 190)$ that was significantly less to it with a copper-copper collector of $(\$ 280)$ each material had comparable performance. [8] in is analysis carried out an investigation on a sunny day in China, the characteristics of group action $\mathrm{Ba}(\mathrm{OH})_{2} \quad 8 \mathrm{H}_{2} \mathrm{O}$ with a melting temperature of $81.8^{\circ} \mathrm{C}$ in associate exhausted tube solar tank. The test was implemented for simple charging operation and simultaneous operation. The results showed that integrating the PCM within the exhausted tube solar tank improved the performance when compared with that in all-glass evacuated tube solar water heater. However, the storage of thermal energy depends heavily on the medium that its hold on. To totally utilize the heat of transformation, unleash throughout melting (discharge period), a PCM should meet the required temperature and heat of transformation necessities of the system. As an example, a solar dish charging a quandary latent TES system wouldn't use water/ice as the PCM, since water is already within the liquid state at room temperature. Likewise, one couldn't use significant metals like copper or iron as a PCM, since the melting temperature is far too high, and wouldn't be accessible in an exceedingly solar dish. It's for this reason that a good variety of PCM materials are studied within the literature.

In this paper, the unsteady heat transfers between the phase change material and the operational fluid was numerically investigated employing a finite distinction theme approach. The operational fluid is water and also the PCM is mixture of eutectic- salts. The convective heat transfer-coefficient is computed by means of empirical relationships as functions of Reynolds number, the Prandtl number and geometry of the heat exchanger. Underneath simplifying assumptions, the governing equation of the problem is the energy conservation equation written for the heat transfer fluid, storage tank and the PCM. The primary equations are discretized using explicit schemes, whereas the secondary is discretized employing a semi-implicit theme. The PCM is analysed by writing the energy conservation equation within the variety of enthalpy of the PCM. The target of this paper is to analyse the unsteady heat transfers between operational fluids (water) and storage tank integrated with PCM. A mathematical model has been developed and the finite distinction methodology is used for the computation of the thermal behavior of the PCMs. A comparison with the experimental results is formed and a number of other simulation runs are conducted for the typical close, inlet, and outlet conditions for parameters of interest.

\section{A. Mathematical Model of a Thermal Storage Tank with PCM}

In this section, a mathematical model of a storage tank with PCM is developed. The PCM tank is broken down into 11 segments of 0.88 lengths in axial direction. Each layer is considered to be a cylinder which has the same thermal behavior, varying its temperature along the radial direction. The simplified model is composed of two sub-models: one describing the PCM behavior in the sensible heat stage and another which describes the PCM dynamics in the phase change stage. The heat transfer processes within the adopted phase transition materials structures are advanced, particularly the substance within the transition stage. The phase transition materials can exist in 3 states in 2 phases: solid, liquid and mushy. To simplify the mathematical model, the subsequent assumptions were made:

1. The adopted composites are treated as a body of uniform equivalent physical and thermal properties primarily specific and heat, density and thermal conduction.

2. The phase transition material is physical property and unvaried in nature.

3. The heat transfer method is thought of in each way (1D, and 2D).

The differential equations of transient heat physical phenomenon with variable thermo-physical properties are given by:

$\frac{\partial}{\partial t} \rho h(T)=\nabla \cdot[\lambda \mathrm{T} \cdot \nabla T(r, t)]+g(r, t)$

Where $T$ are temperature, $\rho$ density, $h$ enthalpy, $\lambda$ conductivity and $g$ heat generation rate.

Where

$\frac{\partial \rho}{\partial t} \approx 0, \frac{\partial h}{\partial t}=\frac{\partial h}{d T} \times \frac{\partial T}{d t}=C_{e f f} T \frac{\partial T}{\partial t}$

Where equation (1) can be further expressed as

$\rho T=C_{\text {eff }} T \frac{T\left(r_{t}, t\right)}{\partial t}=\nabla \cdot\left[\lambda \mathrm{T} \cdot \nabla T\left(r_{,} t\right)\right]+g(r, t)$

The non-linear problem within the PCM range is outlined by equation (3), the Goodman rework [10] will be used to get rid of the temperature dependent, effective heat capability, $C_{\text {eff }}$ outside the differential operator by defining a new dependent variable:

$v=\int_{C_{n_{1} S} C_{1, f f}}^{C_{1}} C_{\text {eff }} T T$

Where $C_{2, S}$ is the heat capacity in solid phase, $C_{1, l}$ the heat capacity in liquid phase and $C_{\text {eff }}$ is the effective heat capacity.

Equation (3) may be additional expressed as:

$\rho T=C_{\text {eff }} T \frac{\partial T}{\partial v} \frac{\partial v}{\partial T}=\nabla \times\left[\lambda \mathrm{T} \times \frac{\partial T}{\partial v} \Delta v+g(r, t)\right](6)$

The phase change problem conjugated to conduction in the storage tank wall and to the forced convection of the heat transfer fluid actually is unsteady [11]. The reason been that solely the physical phenomenon mode of the heat transfer within the activity are thought of and the analysis of the thermal system simplified. Besides with Rayleigh numbers $<$ than $1 \times 10^{6}$ this presumption ends up in a suitable margin of error. Bringing this concession into bear, the governing equations will be written in cylindrical reference systems (storage tank) that embody the momentum balance equation for the heat transfer fluid and also the conservation of mass. In keeping with [12] temperature is therefore a function of the heat content during the phase change. 


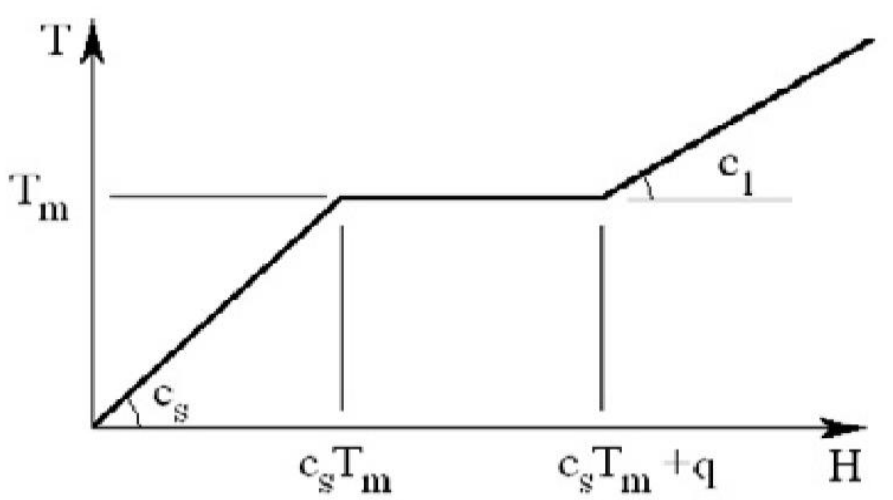

Figure 2. Isothermal phase transition [12]

Determining the state of the phase change materials (Solid liquid undergoing phase change).

According to [12]

The liquid fraction $x=\frac{m^{1}}{m^{\text {total }}}$.

Where;

$m_{\text {total }}=$ Total mass of the phase change material

$m_{s}=$ The mass of the phase change material in the solid state $m_{I}=$ The mass of the phase change material in the liquid state.

The liquid fraction of the phase change can be written as $x=\frac{H \leq c_{s} T_{m}}{q}$.

For $x \leq 0 \quad H \leq C_{g} T_{m}$

For $0<x<1 \quad C_{g} T_{\mathrm{m}}<H<C_{g} T_{\mathrm{m}}+q$

For $x \geq 0 \quad H \geq C_{g} T_{m}+q$

The above equations describe the answer of flow field, heattransfer and phase change at the same time via finite component methodology (FEM) [13] the heat-transfer fluid (HTF) during this case is assumed incompressible and one Dimensional [14]. This presumption implies that it's doable to separate the equation of motion from the equation of energy, (neglecting the continuity and momentum equation of the HTF). It's vital to grasp that the heat exchange mechanism among the heat-transfer fluid is convection. However, it's doable to model the heat-transfer among the HTF through a convenient suggests of the heat transfer constant $h$. Prandtl number $P_{r}=0.727$ and a Reynolds number, $R_{e}$ of 1140 and also the characteristic size $D_{i_{2} \text { tank }}$ of the storage-tank, with this it's doable to calculate the Nusselt number, $N u=1.86\left(\frac{R_{e} P_{Y} D_{i}}{L}\right)^{1 / 3}$.

According to [15] the heat exchange coefficient of the storagetank can be determine as

$h=N u \lambda / D_{1}$

Putting the tube cross section into thought $A_{h t f}$ the conservation energy equation for the heat transfer fluid (HTF) becomes

$\rho_{h t f} c_{h t f} \frac{\partial T_{h t f}}{\partial t}=-\frac{c_{h t f} \tilde{m}_{h t f}}{A_{h t f}} \frac{\partial T_{h t f}}{\partial x}+$

$\frac{h \pi D_{i, \operatorname{tank}}}{A_{h t f}}\left(T_{w(\operatorname{tank})}-T_{h t f}\right)$

Assuming the thermal resistance of the wall is negligible, the conservation energy equation of the wall now becomes:

$$
\begin{aligned}
& \rho_{w} c_{w} \frac{\partial T_{w}}{\partial t}=\frac{h \pi D}{A_{w 1}}\left(T_{h t f}-T_{w(\operatorname{tank})}\right)+\frac{1}{s_{w}}\left(\frac{k_{w} A_{w}^{1}}{A_{w 1}}+\right. \\
& \left.\frac{k_{p c m} A_{p c m}^{1}}{A_{w 1}}\right)\left(T_{p c m}-T_{w(\tan k)}\right)
\end{aligned}
$$

Where:

$k_{w}$ and $k_{p c m}$ are the thermal conductivity of the tank wall and the PCM respectively,

The heat transfer conductivity within the PCM in the axial direction is negligible, thus the conservation energy equation within the 1st cell of the PCM alternate to the wall becomes:

$$
\begin{aligned}
& \rho_{p c m} \frac{\partial H_{p c m}}{\partial t}=\frac{1}{s_{w}}\left(\frac{k_{w} A_{W 11}^{1}}{A_{w 1}}+\right. \\
& \left.\frac{k_{p c m} A_{p c m}^{1}}{A_{w 1}}\right)\left(T_{p c m}-T_{w(\operatorname{tank})}\right)+\frac{1}{s_{w}} \frac{k_{p c m}}{A_{p c m}}\left(\frac{H_{p c m}^{l+1}}{A_{w 1}}-\right. \\
& \left.\frac{H_{p c m}^{j}}{A_{w 1}}\right)
\end{aligned}
$$

The energy conservation equation for the universal cell of the PCM becomes:

$$
\rho_{p c m} \frac{\partial H_{p c m}}{\partial t}=\frac{k_{p c m}}{c_{p c m}}\left(\frac{1}{r} \frac{\partial}{\partial r}\left(r \frac{\partial H_{p c m}}{\partial r}\right)\right)
$$


In [16] a more advanced mathematical model of the PCM storage tank is presented. The mathematical model is based on the Stefan solution for the phase-change stage. Experimentally, from a thermal point of view, there are two types of tube: tubes placed in the central zone, and those placed in the outer most layer of the storage tank. The behavior of the central tubes is considered the same. The percentage of the tubes placed in the outermost zone is very low compared to that placed in the central zone (as shown in Figure 3).

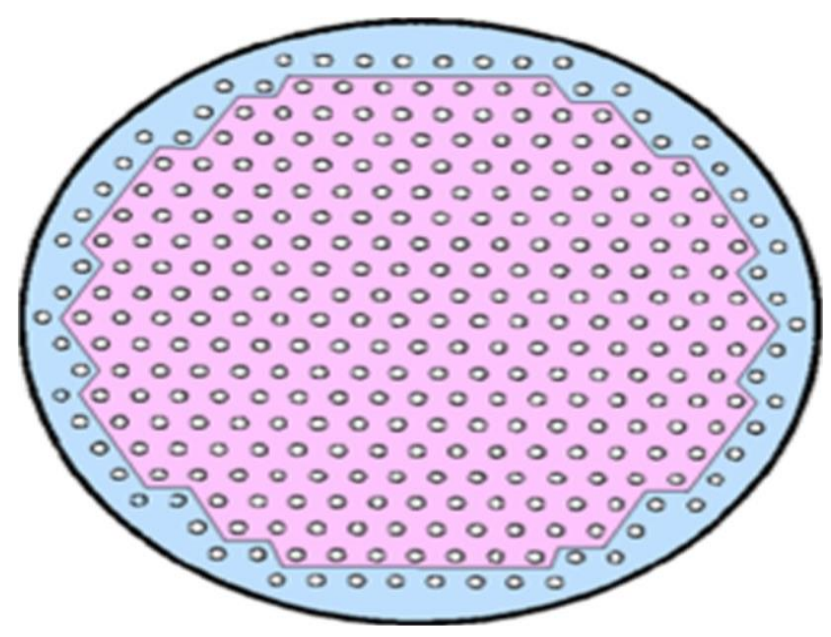

Figure 3. Tube dispersal inside the phase change material tank [16]

In each segment, the temperature is computed via a dual stage process. First, each one is corrected in function of losses produced by the energy transport of the water flow and secondly, they are validated using heat transfer equations, depending on if they are in the sensible heat transmission stage or the phase change stage. To compute the temperature evolution in the sensible heat transfer stage, two energy balance equations are used for the liquid and solid phase. The difference between them lies in their density, specific heat and thermal conductivity. The resistance and capacity of each layer depend on the separation radius. The higher the separation radius the higher the thermal resistance and vice versa. The equations based on Stefan's are used to compute the PCM temperature evolution. The behavior of the PCM is depicted in Figure 4.

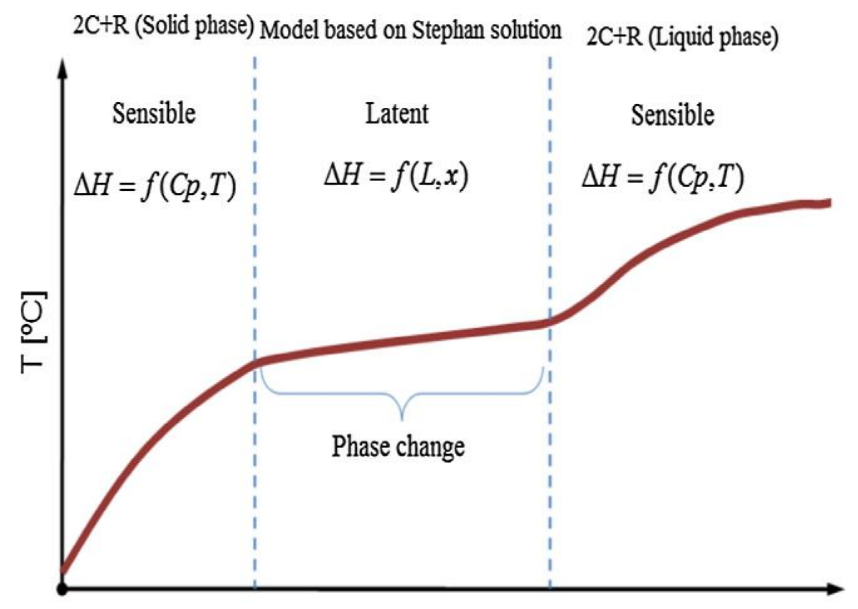

$\mathrm{t}[\mathrm{s}]$

Figure 4. Phases in phase change materials. [16]

At first, the PCM is in the solid phase, when it reaches the melting temperature, the phase change starts. The PCM temperature evolves at an almost constant temperature. Stefan's solution considers that, at the first stage, the whole PCM is at the phase change temperature. As the interface evolves, the temperature of the material phase change does not remain constant. The PCM get melted on reaching the liquid phase. The heat transmission models are described in the following subsections. In order to develop this part of the model, it is necessary to take into account the PCM as shown in Figure 5.

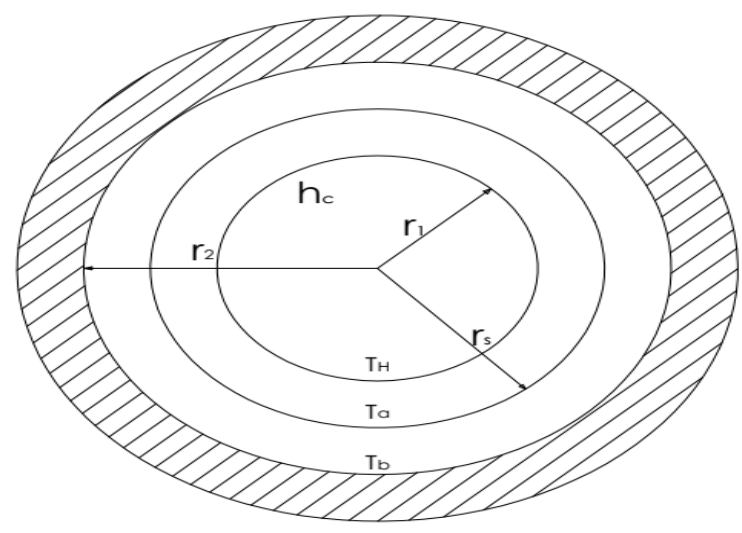

Figure 5. PCM Tank Trigonometry

Adiabatic boundary condition was imposed to external radius of the cylindrical layers due to the symmetric configuration of the storage tank. The $r_{2}$ and $r_{1}$ denote the external and internal radius of the storage tank. $r_{s}$ denote the separationradius dividing the two zones of the $r_{2}$ and $r_{1} . T_{a}$ and $T_{b}$ 
represent temperatures of the zones at $\mathrm{A}$ and $\mathrm{B}$ respectively and $T_{\varphi}$ stands for the hot water temperature.

The differential equations, per zone of the model are as follows:

$$
\begin{aligned}
& \rho C_{p} \pi\left(r_{s}^{2}-r_{1}^{2}\right) \frac{d T_{a}}{d t}=2 \pi r_{1} h\left(T_{\varphi}-T_{a}\right)- \\
& \frac{2 \pi k\left(T_{a-} T_{b}\right)}{\operatorname{In} \frac{\gamma_{2}}{r_{1}}}
\end{aligned}
$$

For the second zone

$\frac{2 \pi k\left(T_{a-} T_{b}\right)}{\operatorname{In} \frac{\pi_{2}}{r_{1}}}=\rho C_{p} \pi\left(r_{2}^{2}-r_{s}^{2}\right) \frac{d T_{b}}{d t}$

It can be seen that the above two equations are identical for each states, however physical properties such as thermalconductivity $(K)$ and density $(\rho)$ have different values because of phase change (solid - liquid-solid states).

\section{B. Phase change based on Stefan solution.}

The phase change starts when the temperature of the PCM exceeds halved temperature of the adopted PCM. In this stage the motion of the adopted PCM is influenced by a phase change. Stefan problems works with an assumption, thus making its inferior compared to the actual energy stored, the assumptions include: solid and liquid phase are assumed stable while the thermal energy storage that comes from the temperature change between the two phases (sensible) are neglected. The use of Stefan number is actually needed to solve for adequacy.

$S_{n}=\frac{C_{p}\left(T_{f}-T r_{1}\right)}{L}$

In this case, the Stefan number approaches zero due to it sensible heat nature which was very small as compared to latent heat. In line with this, Naterer, 2002 proposed a solution equation to remedy the semi-infinite medium of the storage tank and PCM at the initial phase change temperature. The processes of deriving the equation are out of scope in this paper but the final obtained equation is expressed as;

$T(r)=T_{f}+\frac{h_{r 1}}{K} \times\left(\frac{T_{f}-T_{\infty} \times \frac{h_{r 1}}{K} \times \ln \frac{r_{1}}{R}}{1-\frac{h_{r 1}}{K}-\operatorname{In} \frac{r_{11}}{R}}-T_{\infty}\right) \times \operatorname{In} \frac{r}{R}$
$t_{s}=C \times\left(r_{1} \times\left[\frac{h_{r 1}}{K}-2\right]-R^{2}\left(\frac{2 h}{K} \times \ln \frac{r_{1}}{R}+\frac{h}{K}-\right.\right.$ $\left.\frac{2}{r_{1}}\right)$ )

$C=\frac{\rho L^{\tilde{i}}}{4 h\left(T_{\infty}-T_{f}\right.}$

In which $R=R\left(t_{s}\right)$ is the interface position that depends on (Stefan time) time, $T_{f}$ is the melting-temperature, $T(r)$ is the phase change material temperature that depends on the position in radial direction and $L^{1}$ is the corrected heat energy of eutectic mixture. This resolution is valid whereas $R<r_{\theta}$ as a result of the case $R=r_{\theta}$ implies that the full mass has modified its phase .Since one amongst the assumptions of the Stefan resolution is that the full mass is at the melting temperature, the heat-energy should be corrected by adding the required heat to make the full mass be at its melting-temperature. Let $T_{l}$ be the temperature of the last PCM section. The corrected latent-heat is computed as follows:

$L^{L}=m C_{p} \theta$

$m=\pi\left(r_{2}^{2}-r_{1}^{2}\right) \times \rho_{\text {solid }}$

$L^{l}=\pi\left(r_{2}^{2}-r_{1}^{2}\right) \times C_{p} \times \rho_{\text {solid }} \times T_{l}-T_{f}$

\section{Analytic Approximate Solution}

The problem of unsteady compressing of a viscous incompressible fluid delineated by the equation (10-13) between thermal storage vessels in motion is numerically solved using difference scheme. The spatial by-product of the tank is discretized by means of 1 st order forward finite difference scheme and first order backward difference scheme. The discretization of equation (9) associated (10) using forward (Euler) finite difference scheme with time generates an expression for the heat transfer fluid as shown below,

$$
\begin{aligned}
& T_{h t f} \frac{i(m+1-m)}{\Delta t} \cong \frac{1}{\rho_{h t f} c_{h t f}}\left\{\frac{c_{h t f} m_{h} t f}{A_{h t f}} \frac{\partial T_{h t f(m i}}{\partial x}+\right. \\
& \left.\frac{h \pi D_{1}}{A_{h t f}}\left(m, i\left(T_{w(\operatorname{tank})}-T_{h t f}\right)\right)\right\}
\end{aligned}
$$

The temperature of the wall of the thermal storage is expressed as: 


$$
\begin{aligned}
& T_{h t f} \frac{i(m+1-m)}{\Delta t} \cong \frac{h \pi D_{1}}{A_{w} \rho_{w} c_{w}}\left(m, i\left(T_{h t f}-T_{w(\operatorname{tank})}\right)\right)+ \\
& \left.\frac{k_{W} A_{W}^{1}}{A_{w}}+\frac{k_{p c m} A_{p c m}^{1}}{A_{w}}\right) \frac{\left(m_{1} i\left(T_{p c m}-T_{w(t a n k}\right)\right)}{s_{w} \rho_{w} c_{w}}
\end{aligned}
$$

The thermal diffusion in the $j$ index radial direction in the conservation energy equation for the adopted phase change is discretized with respect to centered finite difference scheme with second order accuracy. This discretization from [18-24] results to equation (25)

$$
\begin{aligned}
& H_{p c m} \frac{i(m+1-m)}{\Delta t} \cong \\
& 0.5\left(\frac{1}{\rho_{p c m}} \frac{k_{p c m}}{c_{p c m}}\left\{\frac{\partial}{r . \partial r}\left(r \frac{\partial H_{p c m}}{\partial r}\right)\right\} \wedge^{m+1}+\right. \\
& \frac{1}{\rho_{p c m}} \frac{k_{p c m}}{c_{p c m}}\left\{\frac{\partial}{r \cdot \partial r}\left(r \frac{\partial H_{p c m}}{\partial r}\right)\right\} \wedge^{m}
\end{aligned}
$$

This approximate solution relies on the computation of the values of the fluid and wall temperature at each $i$, node with regard to time as an operation of the values measured at a given time in relation to Euler explicit scheme. The temperature values obtained from this approximate answer are used as boundary conditions for the conservation energy equation within the PCM. It is evident that the worth of the physical property (enthalpy) within the $j$, node depends on the assumed physical property values within the $j-1, \& j+1$ nodes respectively.

\section{III.EXPERIMENT AND RESULT}

The solutions of the mathematical equation facilitate us to work out the temperature profile at every time within the PCM at every point in the tank. The heat transferred from the fluid is absorbed by the PCM through the wall. The obtained radial temperature distribution helps to work out how the thermal gradients are going to be generated within the PCM as a result of time. (Decreases in slope result in a rise in time). The thermal gradient at any purpose depends on the thermal physical phenomenon (thermal conductivity) of the adopted PCM and its thermal inertia. The analysed model during this analysis work is validated using the experimental and simulated results obtained throughout the research conducted within the month of Gregorian calendar month as shown in Figure 6. Within the reason behind the experiment; the measured ambient temperatures vary more or less 27 to $36^{\circ} \mathrm{C}$. In the theoretical analysis, the ambient temperature was kept constant with convective boundary condition on the inner of the PCM thermal storage. Hence, while comparing the theoretical model with the experimental and simulated work, the ambient temperature was kept constant $\left(27^{\circ} \mathrm{C}\right)$ as input within the theoretical as well as the SolidWork simulation software used. The other parameters concerned during this analysis include the likes of inlet and outlet water temperature integrated with PCM, heat transfer coefficients of each of the inner and higher layers of the thermal storage tank, global solar radiation, glass temperature, geometrical, parameters and physical properties of the thermal storage system.

Figure 6 shows the temperature difference between the simulated model and the data obtained in the course of the experimental research work for comparison purposes.

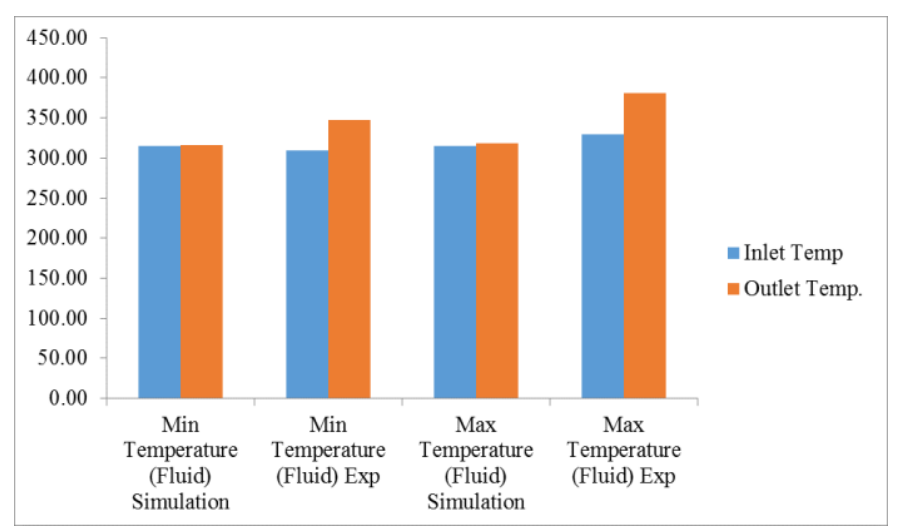

Figure6. Comparison between the simulated and experimental results

A minimum inlet temperature of $41.54^{\circ} \mathrm{C}$ and $36^{\circ} \mathrm{C}$ was observed for both the simulated and experimental model while $42.69^{\circ} \mathrm{C}$ and $74^{\circ} \mathrm{C}$ were both recorded for the minimum outlet temperatures respectively. It was observed from the obtained data that the minimum outlet temperatures of the experimental model outweigh the minimum outlet temperature of the simulated model. The same process was observed for the maximum temperature. A maximum outlet of $108^{\circ} \mathrm{C}$ was obtained from the experimental model compared to an average $45^{\circ} \mathrm{C}$ obtained from simulation. [17-18] carried out studies on storage tank and solar water heater using copper tubes integrated with paraffin wax. The experiment generated a maximum output temperature of $66.6^{\circ} \mathrm{C}$ at the solar peak period and $44.8^{\circ} \mathrm{C}$ the next morning. [18] worked on similar storage tank and solar water heating system and paraffin wax was again used as the PCM with melting temperature of $53.5^{\circ} \mathrm{C}$ the charging results showed that the temperature of the PCM increased at a low temperature gradient owing to the low thermal conduction of the solid PCM. Once the PCM melted, the temperature distinction between layers diminished due to natural convection impact. The discharging results showed that the discharge mass flow and the natural heat transfer constant had a good influence on the storage tank and solar collector performance. However, a definite temperature distinction (experimental) of $61.3^{\circ} \mathrm{C}$ and $54.5^{\circ} \mathrm{C}$ was recorded in comparison to results obtained by each [17-18] and [19]. 
The progression of the graph confirms the efficiency and heat retentive capability of the eutectic metallic phase change materials utilized in this analysis work as compared to paraffin used in the simulation.

\section{CONCLUSION}

In this analysis work, the thermal energy storage between the heat transfer fluid (HTF) and the phase change materials (PCM) was analysed and the temperature field solution of the integrated PCM were obtained numerically through differential equations governing the problem. The HTF and the wall of the energy conservation equation were discretized with an explicit scheme whereas energy conservation equation of the PCM were discretized with semi-implicit scheme. The numerical model proved beyond doubt to be stable and able to proffer solution to problems within a short period of time compare to experimental and simulation work that need an extended waiting period. However, the experimental analysis work indicated that salt hydrates are the foremost energy intensive of the PCM potentialities in comparison to paraffin for thermal energy storage. This assessment additionally brings into lime lightweight that once PCM was used as a medium for energy storage, it considerably accumulates a lot of energy than within the case when water was used as a medium for energy storage. Finally, the speed of getting lead to the numerical model shows computational simplicity and unsteady behavior of the thermal energy storage.

\section{ACKNOWLEDGEMENT}

The authors are grateful to Cecilia Titilope Aduke Isamotu foundation for the moral and financial commitment towards the realization of this research work May God continue to rest your Soul until will meet to part no more.

\section{REFERENCE}

[1] Intergovernmental Panel on Climate Change 2014 Climate Change 2014 - Impacts, Adaptation, and Vulnerability.

[2] Demirbas M.F. (2006) "Thermal Energy Storage and Phase Change Materials: An Overview,

Energy Sources", Part B, 1, (pp. 85-95).

[3] Pop O., Fechete L., and Balan M. (2017) "Numerical model for solidification and melting of PCM encapsulated in spherical shells", Energy Procedia, 112, (pp. 336-343). https://doi.org/10.1016/j.egypro.2017.03.1060

[4]Nussbaumer T., Wakili K.G., and Tanner C. (2006) "Appli ed Experimental and Numerical Investigation of the thermal performance of a protected vacuum- insulation system applied to a concrete wall”, no. 83, (pp. 841-855).

https://doi.org/10.1016/j.apenergy.2005.08.004

[5] Rabin Y., Bar-Niv I., Korin E., and Mikic B. (1995) "Integrated solar collector storage system based on a salthydrate phase change material", Solar Energy, no. 55, (pp. 435-444).
[6] Lin S.C., Al-kayiem H.H., and Aris M.S. (2012) "Experimental investigation on the performance enhancement of integrated PCM-flat plate solar collector". Journal of Applied Sciences. 12(9) (pp. 2390-2396).

[7] Nahar N.M., (2002) "Capital cost and economic viability of thermosyhonic solar water heaters manufactured from alternate materials in India", Renewable Energy no.26, ( pp. 623-635).

[8]Xue H.S. (2016) "Experimental investigation of a domestic solar water heater with solar collector coupled phase-change energy storage", Renewable Energy, no. 86, (pp. 257-261).

[9] Sharma A., Sharma N., Pradhan B., Kumar. (2003) "Performance evaluation of a solar water heater having built in latent heat storage unit", IEA, ECESIA Annex 17. Advanced thermal energy storage through phase change materials and chemical reactions-feasibility studies and demonstration projects. $4^{\text {th }}$ workshop, Indore, India. (pp.109-115).

[10]Samarskii A.A., and Vabishchevivh P.N. (1995)

"Computational Heat Transfer", volume1 Mathematical Modelling. John Wiley and Sons.

[11] Trp A. (2005) "An experimental and numerical investigation of heat transfer during technical grade paraffin melting and solidification in a shell and tube latent thermal energy storage unit," Solar Energy no. 79,(pp. 648-660).

[12]Alawadhi E.M. (2004) " Phase change process with free convection in a circular enclosure: numerical simulations", Computers and Fluid no.33,( pp.1335-1348).

[13]Patanker S.V. (1990) "Numerical heat transfer and Fluid Flow'Hemisphere Publishing Corporation, New York .

[14] Banaszek J., Domanaski R., Rebow M., and El-Sagier F. (2000) "Numerical analysis of the paraffin wax-air spiral thermal energy storage unit", Applied Thermal Engineering, no.20, (pp. 323-354).

[15] Cengel Y."Thermodynamics and Heat trsnsmission", 2012.

[16] Ru1 'z-Pardo A., Salmerón J.M., Cerezuela-Parish A., Gil A. A., lvarez S. and Cabeza L. (2012) "Numerical simulation of a thermal energy storage system with PCM in a shell and tube tank", 12th International Conference on Energy Storage, Innostock.

[17] Khalifa A.J.N., and Abdul Jabbar R.A. (2013) "Conventional versus storage domestic solar hot water systems: A comparative performance study. Energy Conversio n and Management", no.51 (2) (pp.265-270).

[18] Khalifa A.J.N., Suffer K.H., and Mahmoud M.S. (2013) "A storage domestic solar hot water system with a back layer of fluid science", no.44, (pp.174-181).

[19] Mettawee E.B.S., Assassa G.M.R. (2006)" Experimental study of a compact PCM solar collector", Energy, no. 31, (14) (pp. 2622-2632). 